\title{
Clinical Study of Mastoid Abscess - Our Experience
}

\author{
Raies Ahmad Begh, Aditiya Saraf \\ Department of ENT and Head and Neck Surgery, SMGS Hospital, Government Medical College, Jammu, \\ Jammu and Kashmir, India
}

Address for correspondence: Dr Aditiya Saraf; rksaraf56@gmail.com

Received 07 July 2019;

Accepted 30 August 2019;

Published 25 September 2019

\begin{abstract}
Aims and Objectives: To study various aetiologies of mastoid abscess. Material and methods: The present study was conducted in Department of ENT, SMGS Hospital, GMC Jammu from August 2017 to June 2019.A total of 20 patients of chronic otitis media with mastoid abscess were included in the study and were planned for mastoidectomy. In all patients, abscess drainage followed by modified radical mastoidectomy via post-aural approach was done in the same sitting. In all patients, the contents of abscess were sent for histo-pathological, microscopic examination and culture study. Results: Histopathological and microscopic examination of contents of abscess revealed bacterial aetiology (Staphylococcal aureus, Klebseilla pneumoniae) in 11 patients, cholesteatoma in 7 patients and tuberculosis in 2 patients. Conclusion: Mastoid abscess is an important complication of otitis media, requiring prompt diagnosis and intervention.
\end{abstract}

Keywords: mastoid, abscess, cholesteatoma, tuberculosis

\section{Introduction}

The diagnosis of chronic otitis media implies a permanent abnormality of pars tensa or flaccida, most likely a result of earlier acute otitis media, negative middle ear pressure or otitis media with effusion. A number of histopathological changes can develop in the middle ear and mastoid in chronic otitis media. Some changes are direct result of infection and inflammation, while others represent the host response to the disease process. ${ }^{[1]}$

Otitis media is one of the most commonly treated infections in clinical practice today. The majority of patients with otitis media do well with or without anti-microbial therapy. However, there is a subset of patients who develop serious complications from this otherwise self-limiting disease.

Complications can occur in the acute phase of an infection or as result of bony destruction from chronic bio-enzymatic activity. If left unchecked, infection can spread beyond the temporal bone as abscess or intracranially. With the introduction of modern antibiotic therapy, the incidence of such complications has declined dramatically. ${ }^{[2]}$

The management of mastoiditis complicated by an abscess has traditionally required a mastoidectomy irrespective of patient's age. However, in an era of antibiotics, mastoid abscess is an uncommon complication of otitis media now. ${ }^{[3]}$ This has resulted in a decline in the incidence of mastoidectomy performed for mastoid abscess. Also, various treatments for mastoiditis with abscess such as tympanostomy tube insertion and post-auricular incision-drainage have avoided the morbidity and necessity of mastoid surgery. ${ }^{[4]}$

In our study, our aim was to study various aetiologies of mastoid abscess.

\section{Material \& Methods}

The present study was conducted in Department of ENT, SMGS Hospital, GMC Jammu from August 2017 to June 2019

A total of 20 patients of chronic otitis media with mastoid abscess were included in the study and were planned for mastoidectomy.

All patients were subjected to detailed clinical history, general physical examination, local ENT examination including tuning fork tests using 256, 512 and $1024 \mathrm{~Hz}$ frequency tuning forks, pure tone audiometry and EUM (Examination under Microscope).

Routine lab investigations - Complete Blood Count, Renal and Liver Function tests were conducted.

X-Ray Mastoid (Schuller's view) and HRCT Temporal bone was done on all patients.

Informed and written consent was taken from all the patients undergoing mastoidectomy.

In all patients, abscess drainage followed by modified radical mastoidectomy via post-aural approach was done in the same sitting.

In all patients, the contents of abscess were sent for histopathological, microscopic examination and culture study.

All patients were kept in hospital for 8 days post-operatively, followed up every week for first month, then fortnightly for next two months.

\section{Results}

Majority of patients belonged to the age group of 11-20 years, with mean age being 16.6 years. 
Out of 20 patients, 12 were males and 8 were females.

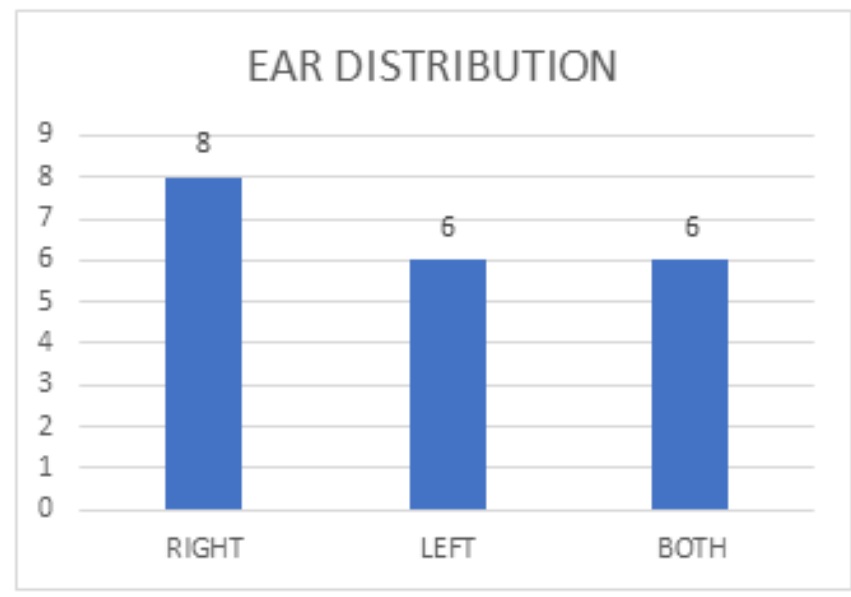

Graph 1

Out of 20 patients, all had pre-existing chronic otitis media; 8 in right ear, 6 in left ear, 6 in both ears.

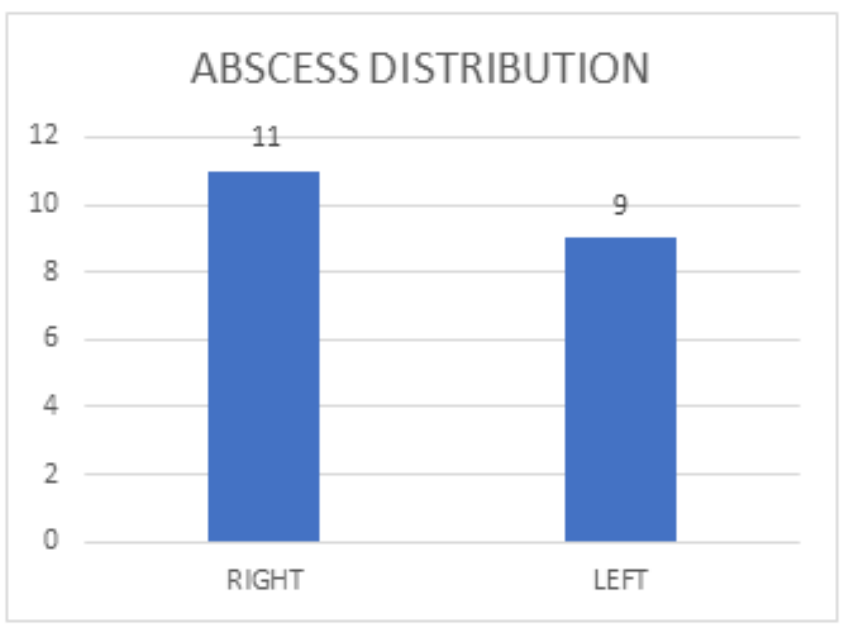

Graph 2

Out of 20 patients, 11 patients had right sided mastoid abscess, 9 had left sided mastoid abscess; none of the subject had bilateral mastoid abcess.

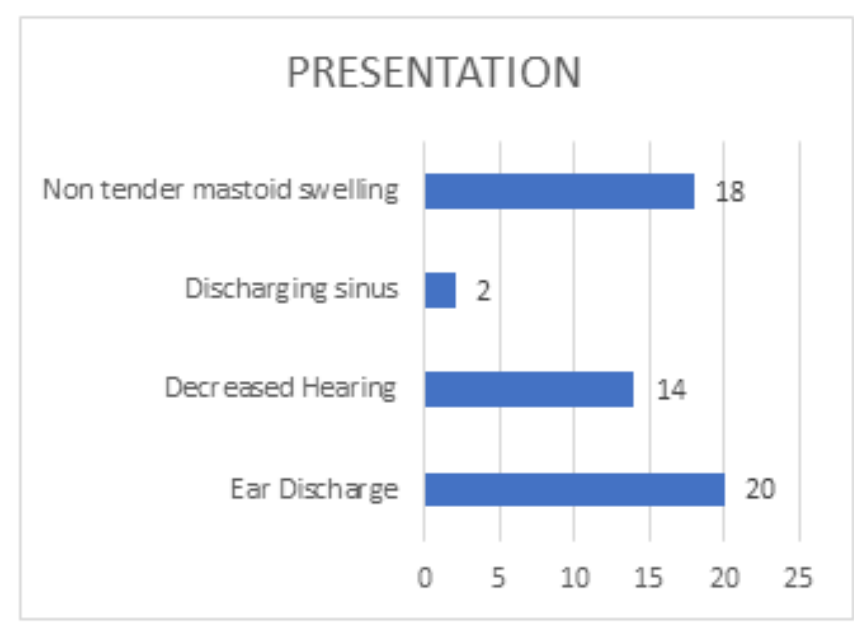

Graph 3

The major presenting complaint was chronic ear discharge (100\%), non-tender mastoid swelling without active discharge (90\%), decreased hearing (70\%), discharging sinus in abscess (10\%).

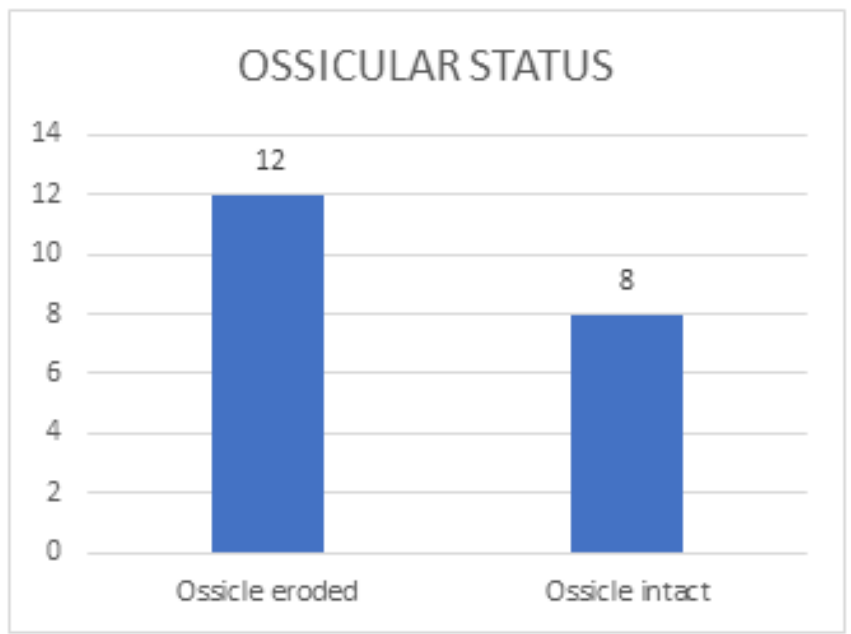

Graph 4

Per-operative Ossicular chain status showed erosion of long process of incus in 10 patients, normal ossicular status in 8 patients and only stapes footplate was present in 2 patients.

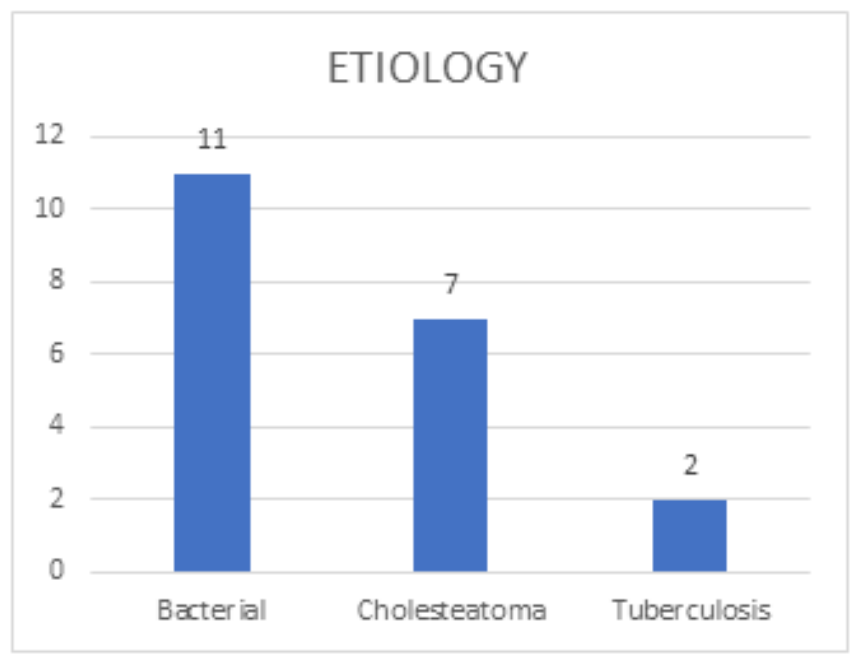

Graph 5

Histopathological and microscopic examination of contents of abscess revealed bacterial etiology (Staphylococcal aureus, Klebseilla pneumoniae) in 11 patients, cholesteatoma in 7 patients and tuberculosis in 2 patients.

\section{Discussion}

Otitis media is an inflammation of part or all of the mucoperiosteal lining of the tympanomastoid compartment comprising the eustachian tube, the tymapanic cavity, the mastoid antrum and all the pneumatised spaces of the temporal bone. ${ }^{[2]}$

Complications of otitis media have been have been defined as spread of infection beyond the confines of the lining mucosa of middle ear cleft. In pre-antibiotic era, $52 \%$ of complications were associated with virulent acute otitis media. Today, the majority of complications result from chronic otitis media. Morbidity and mortality from complications can be minimised if there is a keen familiarity with specific type of draining ear, the underlying pathology and the early signs \& symptoms of complications. ${ }^{[2]}$

The complications of otitis media are broadly categorised into extracranial and intracranial complications. Intratemporal complications include- mastoiditis, petrositis, facial paralysis and labyrinthitis. Intracranial complications include- extradural abscess, subdural abscess, meningitis, brain abscess, lateral sinus thrombophlebitis and otitic hydrocephalus. ${ }^{[5]}$ The contemporary 
risk for developing an extracranial complication of otitis media is approximately twice that for an intracranial complication, with patients experiencing problems such as facial nerve palsy, labyrinthitis, perichondritis or subperiosteal abscess. ${ }^{[6]}$

Mastoiditis has often been recognised as an extracranial complication of otitis media when patients develop post-auricular swelling. An abscess forms between the mastoid cortex and outer periosteum as a result of infection passing from the mastoid air cells or antrum out through the bone. ${ }^{[7]}$ Mastoid abscess occurs when purulent material collection accumulates within the middle ear and mastoid air cells and it is often accompanied by granulation tissue. Surgical intervention is still the most common treatment for mastoid abscess.

Majority of patients belonged to the age group of 11-20 years, with mean age being 16.6 years. Ami et al., ${ }^{[3]}$ in their study showed mean age to be 30.4 years. Raouf MA et al., in their study showed mean age of 11.5 years. ${ }^{[8]}$

Out of 20 patients, 12 were males and 8 were females. Raouf MA et al., ${ }^{[8]}$ in their study showed male predominance.

Out of 20 patients, all had pre-existing chronic otitis media; 8 in right ear, 6 in left ear, 6 in both ears. None of the patient in our study had abscess following acute otitis media.

Out of 20 patients, 11 patients had right sided mastoid abscess, 9 had left sided mastoid abscess; none of the subject had bilateral mastoid abscess.

The major presenting complaint was chronic ear discharge $(100 \%)$, non-tender mastoid swelling without active discharge (90\%), decreased hearing (70\%), discharging sinus in abscess $(10 \%)$. None of the subjects in our study showed any other complication like facial palsy or vertigo. However, in one case intraoperatively a fistula was seen on lateral semi-circular canal although patient was asymptomatic; aetiology of the case came out to be cholesteatoma.

Per-operative Ossicular status showed erosion of long process of incus in 10 patients, normal ossicular status in 8 patients and only stapes footplate was present in 2 patients. The two patients in whom stapes foot plate was the only ossicle present, microscopy showed tuberculosis.

Histopathological and microscopic examination of contents of abscess revealed bacterial aetiology in 11 patients $(55 \%)$, cholesteatoma in 7 patients $(35 \%)$ and tuberculosis in 2 patients(10\%). Raouf MA et al., ${ }^{[8]}$ in their study revealed cholesteatoma in $41.6 \%$ patients. Ami et al., ${ }^{[3]}$ revealed cholesteatoma in $26 \%$ subjects in their study. The two patients who were positive for tuberculosis were sent for a proper ATT course.

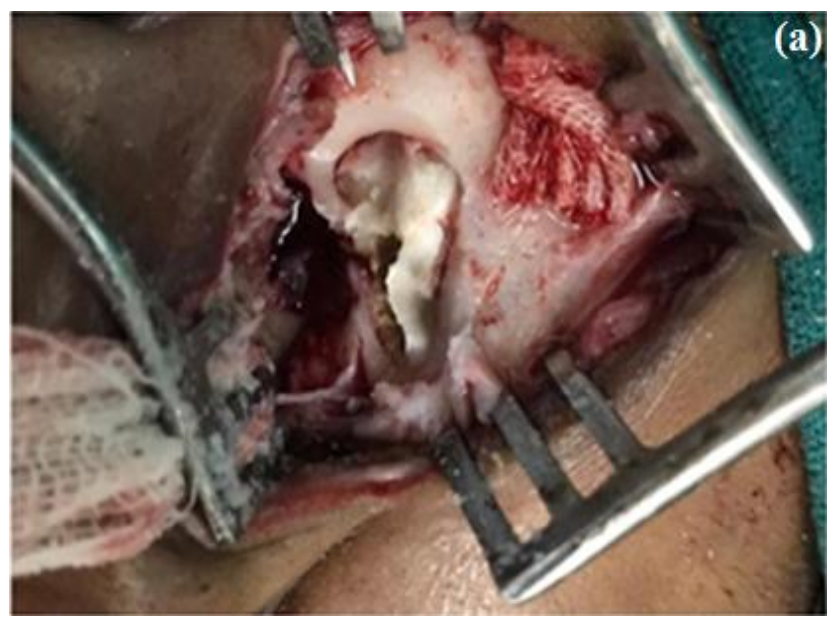

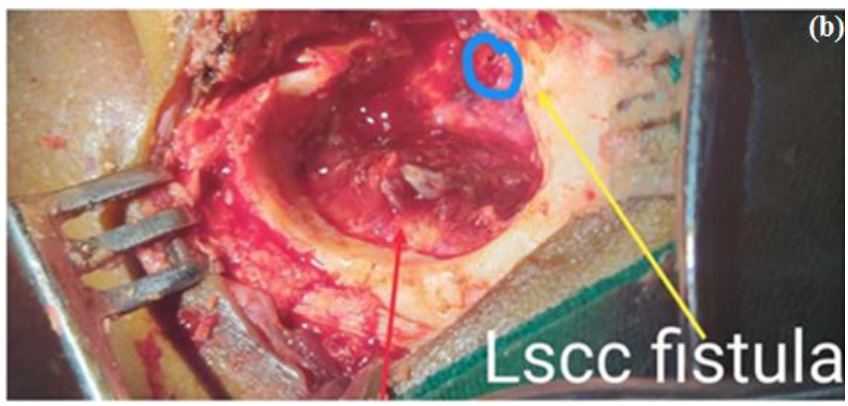

Figure 1: Showing a) Cholesteatoma mass in mastoid b) Mastoid cavity with LSS fistula

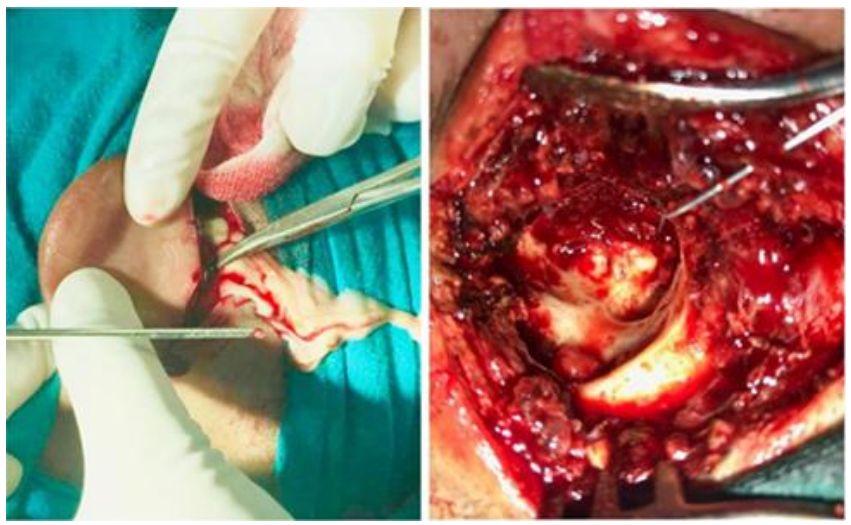

Figure 2: Showing post aural incision and drainage of abcess followed by mastoidectomy

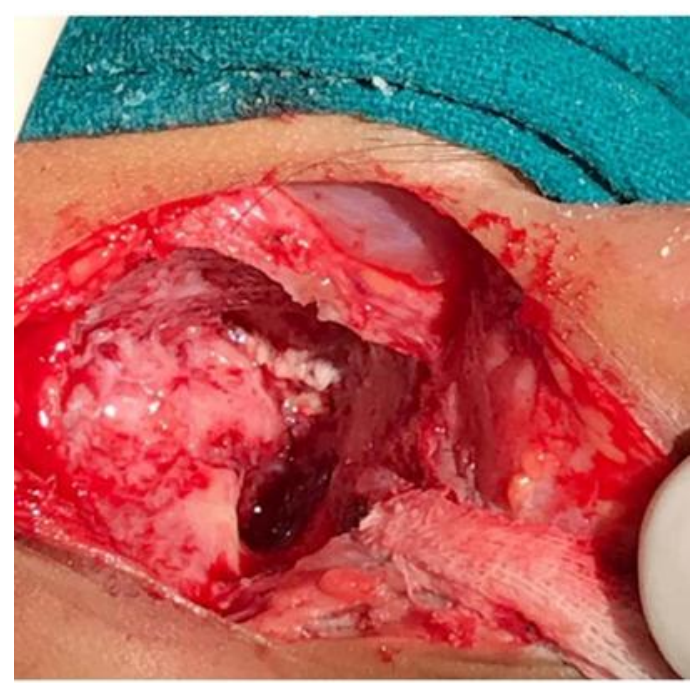

Figure 03: Showing mastoid cavity of patient with tubercular abcess(drained).

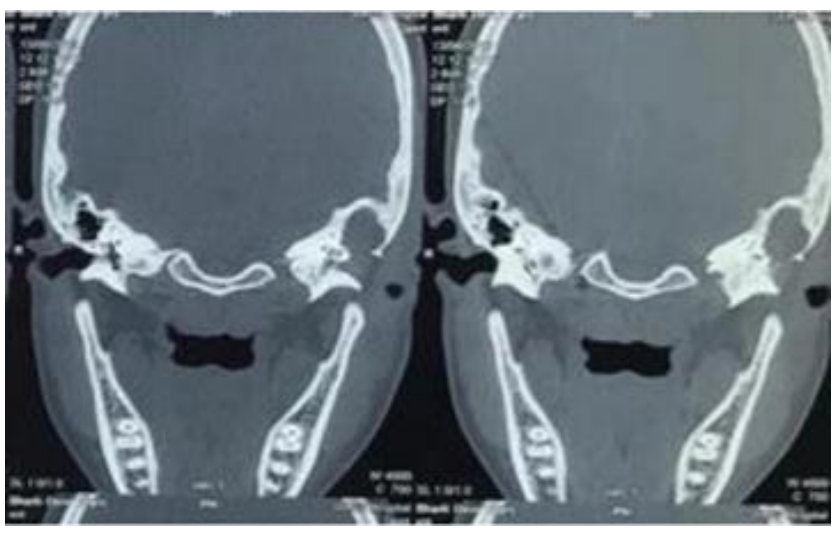

Figure 4: HRCT temporal bone coronal view showing mastoid abscess with erosion of mastoid cortex 


\section{Conclusion}

India is a developing country, with majority of population having limited access to healthcare and do not have proper knowledge of disease and importance of complete the course of antibiotics prescribed. Given the high prevalence of tuberculosis in our country, possibility of tubercular otitis media should also be ruled out. Mastoid abscess is an important complication of otitis media, requiring prompt diagnosis and intervention.

\section{Bibliography}

[1] Scott-Brown's Otorhinolaryngology, Head and Neck Surgery. Volume 3. 2008.Chapter 237c;7/e

[2] Glasscock-Shambaugh Surgery of the Ear, 5th Ed; Chapter 22:pg 435.

[3] Ami Mazita, Zakaria Z, Goh BS, Abdullah A, Saim L. Mastoid abscess in acute and chronic otitis media. Malays J Med Sci. 2010;17(4):44-50.
[4] Kim SR, Choo OS, Park HY. Two cases of Acute Mastoiditis with Subperiosteal Abscesses.Korean J Audiol. 2013;17(2):97-100.

[5] PL Dhingra, Shruti Dhingra, Deeksha Dhingra. Diseases of Ear, Nose and Throat \& Head and Neck Surgery. 2014 6/e.

[6] Spiegel JH, Lustig LR, Lee KC, Murr AH, Schinder RA. Contemporary presentation and management of a spectrum of mastoid abscesses. Laryngoscope. 1998;108:822-828.

[7] Bauer PW, Brown KR, Jones DT. Mastoid subperiosteal abscess management in children. Int $\mathrm{J}$ Pediatr Otorhinolaryngol. 2002;63:185-188.

[8] Raouf MA, Ashour B, Gawad AA. Updated management strategies for mastoiditis and mastoid abscess. Egyptian Journal of Ear, Nose, Throat and Allied Sciences. 2012; 13:43-48. 